\title{
Dilated cardiomyopathy and impaired cardiac hypertrophic response to angiotensin II in mice lacking FGF-2
}

\author{
Corinne Pellieux, ${ }^{1}$ Alessandro Foletti, ${ }^{2}$ Giovanni Peduto, ${ }^{1}$ Jean-François Aubert,${ }^{1}$ \\ Jürg Nussberger, ${ }^{1}$ Friedrich Beermann, ${ }^{2}$ Hans-R. Brunner, ${ }^{1}$ and Thierry Pedrazzini ${ }^{1}$ \\ ${ }^{1}$ Division of Hypertension, University of Lausanne Medical School, Lausanne, Switzerland \\ ${ }^{2}$ Swiss Institute for Experimental Cancer Research, Epalinges, Switzerland \\ Address correspondence to: Thierry Pedrazzini, Division of Hypertension, \\ University of Lausanne Medical School, CH-1011 Lausanne, Switzerland. \\ Phone: 41-21-314-0750; Fax: 41-21-314-0761; E-mail: thierry.pedrazzini@chuv.hospvd.ch. \\ Received for publication June 27, 2001, and accepted in revised form October 19, 2001.
}

\begin{abstract}
FGF-2 has been implicated in the cardiac response to hypertrophic stimuli. Angiotensin II (Ang II) contributes to maintain elevated blood pressure in hypertensive individuals and exerts direct trophic effects on cardiac cells. However, the role of FGF-2 in Ang II-induced cardiac hypertrophy has not been established. Therefore, mice deficient in FGF-2 expression were studied using a model of Ang II-dependent hypertension and cardiac hypertrophy. Echocardiographic measurements show the presence of dilated cardiomyopathy in normotensive mice lacking FGF-2. Moreover, hypertensive mice without FGF-2 developed no compensatory cardiac hypertrophy. In wild-type mice, hypertrophy was associated with a stimulation of the c-Jun $\mathrm{N}$-terminal kinase, the extracellular signal regulated kinase, and the p38 kinase pathways. In contrast, mitogen-activated protein kinase (MAPK) activation was markedly attenuated in FGF-2-deficient mice. In vitro, FGF-2 of fibroblast origin was demonstrated to be essential in the paracrine stimulation of MAPK activation in cardiomyocytes. Indeed, fibroblasts lacking FGF-2 expression have a defective capacity for releasing growth factors to induce hypertrophic responses in cardiomyocytes. Therefore, these results identify the cardiac fibroblast population as a primary integrator of hypertrophic stimuli in the heart, and suggest that FGF-2 is a crucial mediator of cardiac hypertrophy via autocrine/paracrine actions on cardiac cells.
\end{abstract}

J. Clin. Invest. 108:1843-1851 (2001). DOI:10.1172/JCI200113627.

\section{Introduction}

Cardiac hypertrophy represents an adaptive process of the heart in response to work overload, and is common in hypertensive individuals. The renin-angiotensin system, through the activity of angiotensin II (Ang II), is pivotal for blood pressure homeostasis, but can also sustain high blood pressure in patients suffering from hypertension (1). Besides its hemodynamic effects, Ang II directly contributes to cardiac hypertrophy via its growth factor properties $(2,3)$. Along this line, drugs that inhibit Ang II production normalize blood pressure and left ventricular hypertrophy (4). The trophic actions of Ang II result in part from the release of factors with paracrine activities. One of these factors is bFGF, also known as FGF-2 (5). For instance, cardiomyocytes show a better response to Ang II in the presence of cardiac fibroblasts, and this has been attributed to the presence of FGF-2 (6). Accordingly, Ang II has been found to activate FGF-2 expression and release from cardiac myocytes and fibroblasts $(7,8)$. FGF production in the heart has been demonstrated (5), and has been found to be upregulated after cardiac injury (9). Recently, FGF-2 has been implicated in the hypertrophic response to pressure overload (10). In cardiomyocytes, FGF induces phenotypic changes, including the reexpression of genes encoding fetal isoforms of contractile proteins $(11,12)$. However, the mechanisms by which FGF could induce hypertrophy remains unclear. FGF-2 lacks a signal sequence for secretion, suggesting that it may be able to exit the cells only after stretch, injury, or cell death $(13,14)$. Indeed, FGF-2 is released by cardiomyocytes during contraction (13). Moreover, various hypertrophic agonists other than Ang II stimulate the release of FGF-2 $(5,7,15)$.

FGF-2 binds to specific tyrosine kinase receptors, leading to receptor dimerization, which enables the two cytoplasmic domains to cross-phosphorylate each other $(5,16)$. In cardiomyocytes, this receptor stimulates phospholipase $\mathrm{C}$, resulting in the production of diacylglycerol and inositoltriphosphates, and activates protein kinase $\mathrm{C}(16)$. In addition, FGF-2 activates Ras and mitogen-activated protein kinases (MAPKs), namely, the extracellular signal regulated kinases (ERKs), the c-jun $\mathrm{N}$-terminal kinases (JNKs), and the $\mathrm{p} 38$ kinase (17). MAPKs have emerged as prominent players in the development of cardiac hypertrophy $(16,17)$. However, other pathways such as the calcium/calmodulin calcineurin pathway could participate in establishing the hypertrophic phenotype (18).

The two-kidney one-clip (2K1C) model of renovascular hypertension has greatly contributed to our understanding of hypertensive diseases (19). In this model, 
one renal artery is constricted to reduce renal perfusion. This causes plasma renin and Ang II levels to increase rapidly, leading to a chronic elevation of blood pressure and to compensatory cardiac hypertrophy. We recently created mice deficient in FGF-2 expression using homologous recombination in embryonic stem cells. Both high- and low-molecular-weight forms of FGF-2 are lacking in these animals, which appear grossly normal and not different from those described recently by several other groups $(10,20-22)$. In this study, we took advantage of a $2 \mathrm{~K} 1 \mathrm{C}$ murine model (23) and of FGF-2 knockouts to investigate the role of FGF-2 in the development of cardiac hypertrophy.

\section{Methods}

Mice. Mice lacking FGF-2 gene expression (FGF-2/mice) were generated using homologous recombination in embryonic stem cells by replacing most of the second exon, resulting in the deletion of sequences encoding amino acids 82-93 (A. Foletti and F. Beermann, unpublished results). Depending on the strain, mice carry either one or two renin genes (24). C57BL/6 mice are the prototype of one-renin-gene mice. Therefore, to be more relevant to the human situation, mice were backcrossed for five generations in a C57BL/6 background, and males bearing one renin gene were used at 8 weeks of age.

2K1C bypertension. Briefly, mice were anesthetized using $1.5 \%$ halothane in oxygen. The left kidney was exposed, and a clip (0.12-mm opening) was placed on the renal artery, which was isolated by blunt dissection (23). The sham procedure included the entire surgery, with the exception of artery clipping.

Blood pressure. Four weeks after clipping, the right carotid artery was exposed, and a silicon catheter filled with $5 \%$ glucose solution containing heparin $(300$ $\mathrm{IU} / \mathrm{ml}$ ) was inserted into the vessel and tunneled subcutaneously to exit at the back of the neck. Blood pressure was recorded in conscious mice by connecting the catheter to a pressure transducer using a computerized data acquisition system (Notocord Systems, Croissysur-Seine, France).

Echocardiography. Left ventricular dimensions were assessed 4-6 weeks after clipping in anesthetized mice (90 mg/kg ketamine, $5 \mathrm{mg} / \mathrm{kg}$ xylazine, intraperitoneal) by echocardiography using an ATL HDI 5000 ultrasound machine (Philips Medical Systems, Bothell, Washington, USA) equipped with a $12-\mathrm{MHz}$ phase array linear transducer (L12-5). M-mode images were used for measurement of wall thickness, chamber dimension, and shortening fraction.

Cardiac weight index and cardiac protein homogenates. Cardiac weight index was determined as the ratio of cardiac ventricle weight to body weight. Ventricles were homogenized on ice in $1 \mathrm{ml}$ RIPA buffer $(150 \mathrm{mmol} / \mathrm{l} \mathrm{NaCl}$, $0.25 \%$ deoxycholic acid, $1 \% \mathrm{NP}-40,1 \mathrm{mmol} / \mathrm{l} \mathrm{NaVO}_{3}, 1$ $\mathrm{mmol} / 1 \mathrm{NaF}, 1 \mu \mathrm{g} / \mathrm{ml}$ aprotinin, $1 \mathrm{mmol} / \mathrm{l}$ PMSF, 1 $\mu \mathrm{g} / \mathrm{ml}$ pepstatin $\mathrm{A}, 1 \mu \mathrm{g} / \mathrm{ml}$ leupeptin, $1 \mathrm{mmol} / \mathrm{l}$ EDTA, and $50 \mathrm{mmol} / \mathrm{l}$ Tris, $\mathrm{pH}$ 7.5).
Histology. Heart sections from 2K1C wild-type $\left(\mathrm{FGF}^{+/+}\right)$mice and $\mathrm{FGF}-2^{-/-}$mice were stained with hematoxylin and eosin.

Plasma renin concentration. Plasma renin concentrations were measured as described (23). Aliquots of plasma samples were incubated for 15 minutes at $37^{\circ} \mathrm{C}$ in the presence of plasma from nephrectomized rats as a source of renin substrate. The Ang I concentrations produced during the incubation period were determined with a sensitive radioimmunoassay.

Northern blot analysis. Total RNA, purified from frozen tissues using TriPure (Roche Diagnostics Corp., Basel, Switzerland), was subjected to Northern blot analysis. Ten micrograms of total RNA was separated on 1\% agarose-formaldehyde gel, transferred to GeneScreen nylon membrane (NEN Life Science Products Inc., Boston, Massachusetts, USA), and hybridized with radiolabeled cDNA probes spanning the mouse renin (kidney RNA) and atrial natriuretic factor (cardiac RNA) sequences. Signals were quantified using an Instant Imager (Packard Bioscience Co., Meriden, Connecticut, USA), and normalized to the GAPDH signal.

Cell culture. Neonatal ventricles were digested at $37^{\circ} \mathrm{C}$ in $116 \mathrm{mmol} / \mathrm{l} \mathrm{NaCl}, 1 \mathrm{mmol} / \mathrm{l} \mathrm{NaH}{ }_{2} \mathrm{PO}_{4}, 5.4 \mathrm{mmol} / \mathrm{l}$ $\mathrm{KCl}, 0.8 \mathrm{mmol} / \mathrm{l} \mathrm{MgSO}{ }_{4} \cdot 7 \mathrm{H}_{2} \mathrm{O}, 5.5 \mathrm{mmol} / 1$ glucose, and $20 \mathrm{mmol} / 1 \mathrm{HEPES}$ ( $\mathrm{pH} 7.4$ ) containing $0.45 \mathrm{mg} / \mathrm{ml}$ collagenase (Worthington Biochemical Corp., Lakewood, New Jersey, USA) and $1 \mathrm{mg} / \mathrm{ml}$ pancreatin (Life Technologies Inc., San Diego, California, USA). Cells were washed by centrifugation in a 3:1 mixture of DMEM and Medium 199 (Life Technologies Inc.) supplemented with $10 \%$ horse serum (Serotec Ltd., Oxford, United Kingdom), 5\% FBS (Serotec Ltd.), 2 mmol/1 Lglutamine (Life Technologies Inc.), $10 \mathrm{mg} / \mathrm{ml}$ streptomycin, and $100 \mathrm{UI} / \mathrm{ml}$ penicillin (Life Technologies Inc.). Cardiomyocytes and nonmyocyte cells (NMCs) were separated by differential plating (45 minutes), and plated at a density of $1 \times 10^{5} \mathrm{cells} / \mathrm{cm}^{2}$ on gelatin-coated wells and noncoated wells, respectively. After 24 hours, cardiomyocytes were switched to serum-free medium. NMCs were passaged once before switching to serum-free medium. Cells were stimulated with Ang II, FGF-2, or NMC supernatants, washed with PBS, and lysed in $2 \times$ SDS-PAGE loading buffer.

Production of conditioned medium from NMC culture. NMCs were grown to subconfluence in $10-\mathrm{cm}$ dishes, placed in serum-free medium for 24 hours, and stimulated for 24 hours with $100 \mathrm{nM}$ Ang II. FGF-2 concentrations in supernatants were measured by ELISA (Chemicon International Inc., Temecula, California, USA).

MAPK activation. Thirty micrograms of soluble protein was run in a $10 \%$ SDS-PAGE gel and transferred onto nitrocellulose (ECL nitrocellulose; Amersham Pharmacia Biotech Ltd., Little Chalfont, United Kingdom). Phosphorylation of MAPK was determined by incubation with specific antibodies for the phosphorylated and nonphosphorylated forms of p38, ERK, and JNK (New England Biolabs, Beverly, Massachusetts, USA) at $4^{\circ} \mathrm{C}$ in blotto $(20 \mathrm{mmol} / \mathrm{l}$ Tris, $\mathrm{pH} 7.6$, containing $0.1 \%$ Tween- 
Table 1

Cardiac parameters in normotensive and hypertensive FGF-2 ${ }^{+/+}$and FGF-2 ${ }^{-/-}$mice

\begin{tabular}{|c|c|c|c|c|c|c|c|c|}
\hline Group & $\begin{array}{c}\text { Age } \\
\text { (weeks) }\end{array}$ & Genotype & $n$ & $\begin{array}{l}\text { BW } \\
(\mathrm{g})\end{array}$ & $\begin{array}{c}\mathrm{MBP} \\
(\mathrm{mmHg})\end{array}$ & $\begin{array}{l}\mathrm{HW} \\
(\mathrm{mg})\end{array}$ & $\begin{array}{c}\mathrm{CWI} \\
(\mathrm{mg} / \mathrm{g})\end{array}$ & $\begin{array}{c}\text { ANF/GAPDH } \\
\text { ratio }\end{array}$ \\
\hline \multirow[t]{2}{*}{ Sham } & 12 & FGF-2 ${ }^{+/+}$ & 15 & $25.7 \pm 0.7$ & $118 \pm 2$ & $109 \pm 3$ & $4.24 \pm 0.07$ & $92 \pm 7$ \\
\hline & & FGF-2-/- & 17 & $24.9 \pm 1.1$ & $112 \pm 3$ & $100 \pm 5$ & $3.99 \pm 0.09$ & $80 \pm 3$ \\
\hline \multirow[t]{2}{*}{$2 \mathrm{~K} 1 \mathrm{C}$} & 12 & FGF-2 ${ }^{+/+}$ & 13 & $26.8 \pm 0.5$ & $143 \pm 3^{*}$ & $124 \pm 3^{*}$ & $4.62 \pm 0.09^{*}$ & $117 \pm 11^{*}$ \\
\hline & & FGF-2-/- & 17 & $26.2 \pm 0.7$ & $131 \pm 2^{*+}$ & $109 \pm 4^{\dagger}$ & $4.16 \pm 0.08^{\dagger}$ & $87 \pm 5^{\dagger}$ \\
\hline
\end{tabular}

BW, body weight; MBP, mean blood pressure; HW, heart weight; CWI, cardiac weight index; ANF, atrial natriuretic factor. ${ }^{*} P<0.05$ compared with shamoperated animals. ${ }^{\dagger} P<0.05$ compared with $2 \mathrm{~K} 1 \mathrm{C}$ FGF- $2^{+/+}$mice.

20 and 5\% dry milk). Primary antibodies were revealed with anti-rabbit secondary antibodies conjugated to horseradish peroxidase, using a chemiluminescent detection system (Amersham Pharmacia Biotech Ltd.).

Immunofluorescence. Sarcomeres in cardiomyocytes (fixed with $3 \%$ paraformaldehyde) were detected using a monoclonal anti-myomesin B4 antibody kindly provided by Jean-Claude Perriard (Swiss Institute of Technology, Zurich, Switzerland) (25), and a confocal unit equipped with an inverted microscope (LSM 410; Carl Zeiss, Jena, Germany).

Protein synthesis. Protein synthesis was determined by adding $\left[{ }^{3} \mathrm{H}\right]$ phenylalanine $(5 \mu \mathrm{Ci} / \mathrm{ml})$ to culture medium, and counting uptake using standard scintillation techniques.

Expression of $\alpha$-skeletal actin. Protein was detected by Western blot analysis using an anti- $\alpha$-skeletal actin polyclonal antiserum as described (26).

Statistical analysis. Data are expressed as mean \pm SEM. Statistical analysis was performed using the StudentNewman-Keuls test. $P$ values less than 0.05 were considered significant.

\section{Results}

Dilated cardiomyopathy and absence of bypertrophic responses in FGF-2-deficient mice. Using echocardiography, cardiac dimensions and functions were evaluated in mice lacking FGF-2 under basal conditions, or in an Ang II-dependent model of renovascular hypertension and cardiac hypertrophy (the 2K1C model) (Tables 1 and 2). Left ventricular wall thickness was not different between sham-operated FGF-2 $2^{+/+}$and FGF-2-/- mice. However, FGF-2 $2^{-/-}$mice had enlarged left chambers, as shown by the increased diastolic and systolic diameters, and expe- rienced cardiac dysfunction, as shown by the smaller shortening fraction (Table 2). The absence of FGF-2 led to a slight (and not significant) decrease in blood pressure compared with wild-type mice (Table 1). However, both FGF-2 ${ }^{+/+}$and FGF-2-/- animals demonstrated a significant and similar elevation of blood pressure 4 weeks after clipping. Accordingly, renin expression in the kidneys was significantly stimulated in both hypertensive groups (ratio of kidney renin to GAPDH: $68 \pm 8$ vs. 149 \pm 9 in sham-operated and $2 \mathrm{~K} 1 \mathrm{C} \mathrm{FGF}-2^{+/+}$mice, and $68 \pm 4$ vs. $127 \pm 7$ in sham-operated and $2 \mathrm{~K} 1 \mathrm{C} \mathrm{FGF-2-/-}$ mice, respectively; $P<0.05)$. Moreover, circulating renin concentrations were similar in both clipped groups (plasma renin concentrations: $1602 \pm 272 \mathrm{ng} \mathrm{Ang} \mathrm{I/ml/h}$ vs. $1224 \pm 163 \mathrm{ng}$ Ang I/ml/h in $2 \mathrm{~K} 1 \mathrm{C} \mathrm{FGF}-2^{+/+}$mice and FGF-2-/- mice, respectively; no significant difference). We then investigated the role of FGF- 2 in the development of cardiac hypertrophy in this model. Hypertensive FGF-2 ${ }^{+/+}$mice showed a marked increase in cardiac mass (Table 1). Cardiac hypertrophy in response to chronic hypertension was also evident in $\mathrm{FGF}-2^{+/+}$mice by echocardiography, as shown by the increase in wall thickness (Table 2 ). In contrast, hypertensive FGF-2 ${ }^{-/-}$mice showed no cardiac hypertrophic response, as demonstrated by the inability of the heart to increase its mass (Table 1), the absence of a compensatory increase in wall thickness, and no improvement of cardiac dilation (Table 2). The absence of compensatory hypertrophy in hypertensive FGF-2 knockouts is also evident from histological sections of left ventricular myocytes (Figure 1). Furthermore, cardiac performance in hypertensive FGF-2-/- mice, as assessed by the measurement of the shortening fraction, never reached that seen in controls (Table 2). In agreement with this observation, atrial

Table 2

Echocardiographic parameters in normotensive and hypertensive FGF-2 ${ }^{+/+}$and FGF-2-deficient mice

\begin{tabular}{|c|c|c|c|c|c|c|c|c|c|}
\hline Group & Genotype & $n$ & $\begin{array}{l}\text { LVDD } \\
(\mathrm{cm})\end{array}$ & $\begin{array}{l}\text { LVDS } \\
(\mathrm{cm})\end{array}$ & $\begin{array}{l}\text { LVWTD } \\
(\mathrm{cm})\end{array}$ & $\begin{array}{l}\text { LVWTS } \\
(\mathrm{cm})\end{array}$ & $\begin{array}{l}\text { SWTD } \\
(\mathrm{cm})\end{array}$ & $\begin{array}{l}\text { SWTS } \\
(\mathrm{cm})\end{array}$ & $\begin{array}{l}\text { SF } \\
(\%)\end{array}$ \\
\hline \multirow[t]{2}{*}{ Sham } & FGF-2 $2^{+/+}$ & 8 & $0.31 \pm 0.03$ & $0.19 \pm 0.03$ & $0.10 \pm 0.01$ & $0.12 \pm 0.02$ & $0.09 \pm 0.02$ & $0.11 \pm 0.02$ & $39 \pm 2$ \\
\hline & FGF-2-/- & 8 & $0.39 \pm 0.01^{\dagger}$ & $0.29 \pm 0.03^{\dagger}$ & $0.09 \pm 0.02$ & $0.11 \pm 0.02$ & $0.06 \pm 0.02$ & $0.09 \pm 0.02$ & $26 \pm 4^{\dagger}$ \\
\hline \multirow[t]{2}{*}{$2 \mathrm{~K} 1 \mathrm{C}$} & FGF-2 $2^{+/+}$ & 9 & $0.28 \pm 0.02$ & $0.17 \pm 0.02$ & $0.14 \pm 0.02 *$ & $0.16 \pm 0.03 *$ & $0.11 \pm 0.02$ & $0.14 \pm 0.03 *$ & $42 \pm 2$ \\
\hline & FGF-2-/- & 9 & $0.41 \pm 0.02^{\dagger}$ & $0.30 \pm 0.03^{\dagger}$ & $0.07 \pm 0.01^{\dagger}$ & $0.10 \pm 0.02^{\dagger}$ & $0.06 \pm 0.01^{\dagger}$ & $0.08 \pm 0.01^{\dagger}$ & $27 \pm 2^{\dagger}$ \\
\hline
\end{tabular}

LVDD, left ventricular diameter, end-diastole; LVDS, left ventricular diameter, end-systole; LWWTD, left ventricular wall thickness, end-diastole; LVWTS, left ventricular wall thickness, end-systole; SWTD, septal wall thickness, end-diastole; SWTS, septal wall thickness, end-systole; SF, shortening fraction. ${ }^{*} P<0.05$ compared with sham-operated mice. ${ }^{\dagger} P<0.05$ compared with FGF- $2^{+/+}$mice. 

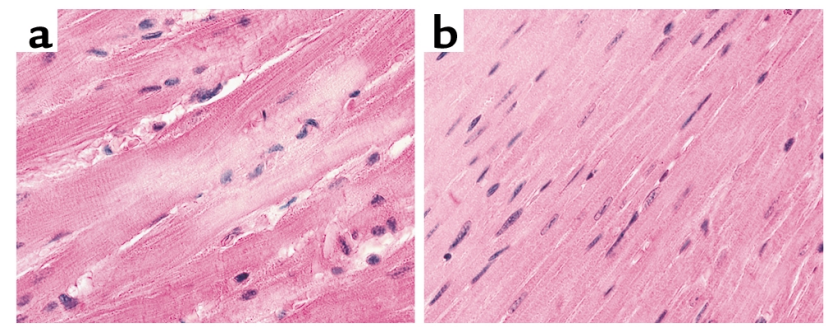

Figure 1

Defective cardiac hypertrophic response to Ang II-dependent renovascular hypertension in FGF-2/- mice. Histological sections of hearts from equally hypertensive FGF- $2^{+/+}(\mathbf{a})$ and FGF-2 ${ }^{-/-}$(b) mice 4 weeks after clipping. See Table 1 for details of the parameters in $2 \mathrm{~K} 1 \mathrm{C}$ mice.

natriuretic factor expression in the hearts of hypertensive FGF-2 $2^{-/-}$mice was not activated (Table 1 ).

Reduced cardiac MAPK activation in the hearts of hypertensive FGF-2-deficient mice. Activation of MAPK has been associated with the development of cardiac hypertrophy induced by growth factors and hemodynamic changes. To determine the role of FGF-2 in MAPK activation during the development of cardiac hypertrophy, phosphorylation of JNK, ERK, and p38 was studied in $2 \mathrm{~K} 1 \mathrm{C}$ mice. All three kinases are activated in the hypertrophied hearts of hypertensive FGF-2 $2^{+/}$controls (Figure 2). In contrast, stimulation of JNK and ERK was reduced in $2 \mathrm{~K} 1 \mathrm{C}$ FGF-2 $2^{--}$mice. Although slightly reduced, activation of $\mathrm{p} 38$ was not significantly diminished in this series of experiments.
Direct MAPK activation by Ang II or FGF-2 in cardiomyocytes is not affected by FGF-2 deficiency. To determine whether the lack of FGF-2 could play a role in the direct stimulation of cardiomyocytes by Ang II or FGF-2, we studied MAPK activation in cardiac myocytes in vitro. Ang II was readily able to activate JNK, ERK, and p38 in cardiomyocytes from either $\mathrm{FGF}-2^{+/+}$mice or FGF-2 ${ }^{-/-}$mice (Figure 3). Similarly, added FGF-2 induced MAPK activation in cardiomyocytes regardless of the capacity of the cells to produced FGF-2.

FGF-2 and FGF-2-dependent trophic factors from NMCs mediate Ang II-induced hypertrophic responses in cardiomyocytes. Since FGF-2 $2^{+/+}$and FGF-2-/- cardiomyocytes appeared to respond normally to Ang II and FGF-2, we investigated possible paracrine stimulation of cardiomyocytes by factors from NMCs. Interestingly, in wild-type cells, FGF-2 seemed to be produced mainly by NMCs (Table 3). Moreover, FGF-2 secretion appeared to be further stimulated by Ang II. The absence of FGF-2 in supernatants from $\mathrm{FGF}-2^{-/}$cardiomyocytes and NMCs was also confirmed (Table 3). Next, conditioned supernatants from NMC cultures were assayed for their capacity to stimulate MAPK in cardiomyocytes (Figure 4). Phosphorylation of JNK, ERK, and p38 was stimulated in FGF-2 $2^{+/+}$ cardiomyocytes by supernatants from $\mathrm{FGF}-2^{+/+} \mathrm{NMC}$ cultures. The stimulatory activity of $\mathrm{FGF}-2^{+/+} \mathrm{NMC}$ supernatants was greatly enhanced when NMCs were stimulated with Ang II. Furthermore, costimulation of FGF-2 $2^{+/+}$cardiomyocytes by FGF-2 ${ }^{+/+} \mathrm{NMC}$ supernatants and Ang II produced maximal activation of the MAPK
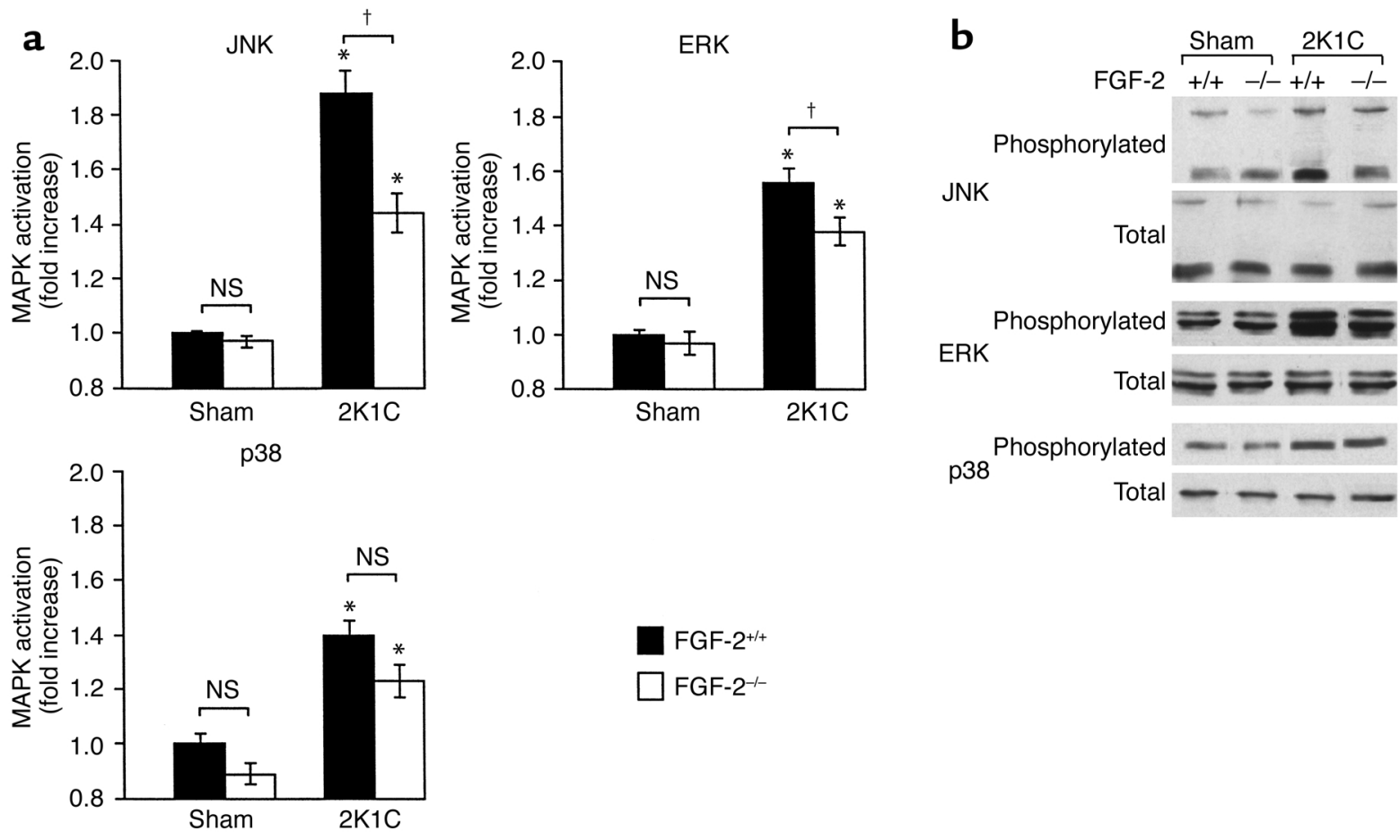

$\mathrm{FGF}^{-2^{+/+}}$

FGF-2 ${ }^{-1}$

Figure 2

MAPK activation in the hearts of normotensive sham-operated (sham) or hypertensive 2K1C mice (a). Phosphorylation of JNK, ERK, and p38 was detected using specific antibodies (b). Data were quantified by laser scanning densitometry after Western blotting. Results represent fold activation compared with sham-operated FGF- $2^{+/+}$controls. Values are expressed as mean \pm SEM $(n=5-10)$. ${ }^{*} P<0.05$ compared with shamoperated $\mathrm{FGF}-2^{+/+}$mice. ${ }^{\dagger} P<0.05$. NS, not significant. 


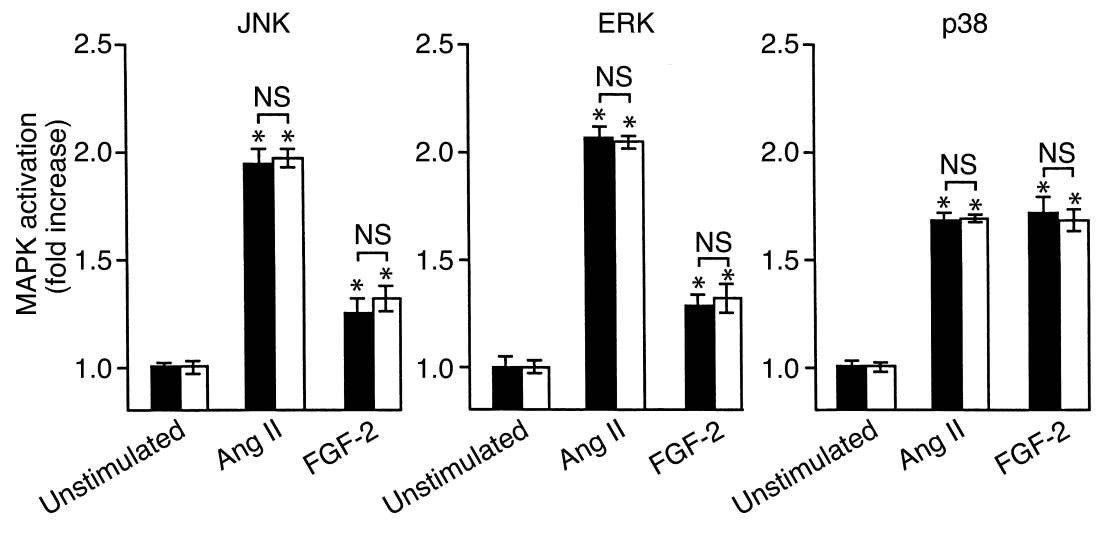

\section{Figure 3}

Normal MAPK activation by Ang II and FGF- 2 in primary cardiomyocytes. Cardiomyocytes from either FGF-2 ${ }^{+/+}$or FGF-2 $2^{-/}$mice were stimulated by either $100 \mathrm{nM}$ Ang II or 20 nM FGF-2 for 10 minutes (JNK) or 5 minutes (ERK and p38). Phosphorylation of JNK, ERK, and p38 was detected using specific antibodies. Data were quantified by laser scanning densitometry. Results represent fold activation compared with unstimulated FGF-2 ${ }^{+/+}$cardiomyocytes. Values are expressed as mean $\pm \operatorname{SEM}(n=3) .{ }^{*} P<0.05$ compared with unstimulated $\mathrm{FGF}-2^{+/+}$cardiomyocytes. NS, not significant. pathways. In sharp contrast, the stimulatory activity of supernatants from FGF-2 $2^{-/-}$NMC cultures was greatly reduced, but not completely abolished.

To further investigate the role of FGF- 2 in the paracrine activation of cardiomyocytes, supernatants from wild-type cultures were tested for their capacity to stimulate MAPK in FGF-2 ${ }^{+/+}$cardiomyocytes in the absence or presence of neutralizing anti-FGF-2 antibodies (Figure 4). Interestingly, the blocking of FGF-2 activity only partially abolished the ability of FGF-2 $2^{+/+}$ NMC supernatants to activate MAPK. Moreover, the capacity of supernatants from cultures containing anti-FGF-2 antibodies to stimulate MAPK was reduced to a lesser extent than that of supernatants from cultures of FGF-2-/- NMCs. This is particularly evident in the case of the JNK pathway.

To exclude a possible inability of FGF-2/-- cardiomyocytes to respond normally to factors from NMCs, we first determined the capacity of cardiac myocytes from FGF-2 knockouts to show a hypertrophic response in the presence of conditioned medium from Ang II-stimulated wild-type NMC culture. As shown in Figure 5, supernatants from FGF-2+/+ NMCs triggered hypertrophy similarly in cardiomyocytes from $\mathrm{FGF}-2^{+/+}$and FGF-2 $2^{-/}$mice, as assessed by measuring the increases in protein synthesis, cell surface area, and sarcomere organization. In contrast, data in Figure 6 demonstrate the reduced capacity of supernatants from NMCs to induce a hypertrophic response in wild-type cardiomyocytes. This inability to induce morphological changes in cardiomyocytes by supernatants from NMCs lacking FGF- 2 was also confirmed by the lack of $\alpha$-skeletal actin expression in myocytes (Figure 6).

\section{Discussion}

The pathways leading to compensatory cardiac hypertrophy remain poorly understood. In the present study, we took advantage of a physiological model of renovascular hypertension to study the role of FGF- 2 in the development of Ang II-dependent cardiac hypertrophy. Mice deficient for FGF-2 expression demonstrated dilated cardiomyopathy, and failed to increase their cardiac mass in response to an elevation of blood pressure. In addition, MAPK activation in the hearts of
FGF-2-deficient mice also appeared to be reduced. This defective MAPK activation results in part from the absence of paracrine stimulation of cardiomyocytes by growth factors from Ang II-stimulated cardiac NMCs - an absence that prevents cardiomyocytes from initiating morphological adaptation to stress.

The FGF family contains about twenty members, which bind to four distinct receptor tyrosine kinases (5). Despite the fact that most FGFs bind to all receptors, each FGF appears to display specific effects. In particular, FGF-2 mediates various biological responses, including mitogenesis and differentiation. FGF-2 is expressed in the heart, and its expression is upregulated by hemodynamic load $(13,14)$. It has been shown to activate MAPK pathways (17), and to play a direct role in the hypertrophic response of cardiomyocytes $(27,28)$. Indeed, the MAPK stimulatory activity of supernatants from fibroblast cultures was significantly attenuated by anti-FGF-2 neutralizing antibodies (Figure 4), suggesting that part of the activity is due to the presence of FGF-2 in these supernatants. This is equally demonstrated by the large decrease in the capacity of supernatants from FGF-2-/fibroblasts to stimulate MAPK (Figure 4). This is in accordance with results showing that pericardial fluid has trophic effects on adult cardiomyocytes, mainly due to high FGF-2 concentrations (28). However, our study also shows that other growth factors are likely to be involved. Indeed, supernatants from FGF-2-/cultures retained some capacity to stimulate MAPK in cardiomyocytes, and anti-FGF-2 antibodies, although in great excess, do not completely abolish the stimulatory activity of culture supernatants (Figure 4).

\section{Table 3}

FGF-2 concentrations $(\mathrm{ng} / \mathrm{ml})$ in supernatants from unstimulated or Ang II-stimulated cardiomyocyte (CM) and NMC cultures

\begin{tabular}{lcccc}
\hline & \multicolumn{2}{c}{ CM } & \multicolumn{2}{c}{$\mathrm{NMC}$} \\
& $\mathrm{FGF}^{+2^{+/}}$ & $\mathrm{FGF}^{-2^{--}}$ & $\mathrm{FGF}^{+/+}$ & $\mathrm{FGF}^{-2^{-/}}$ \\
No Ang II & $0 \pm 0.2$ & $0 \pm 0.6$ & $9 \pm 0.9^{*}$ & $0 \pm 0.5$ \\
100 nM Ang II & $2.9 \pm 0.3^{*}$ & $0 \pm 0.4$ & $18.5 \pm 0.7^{*}$ & $0 \pm 0.3$
\end{tabular}

${ }^{*} P<0.05$ compared with culture medium. 

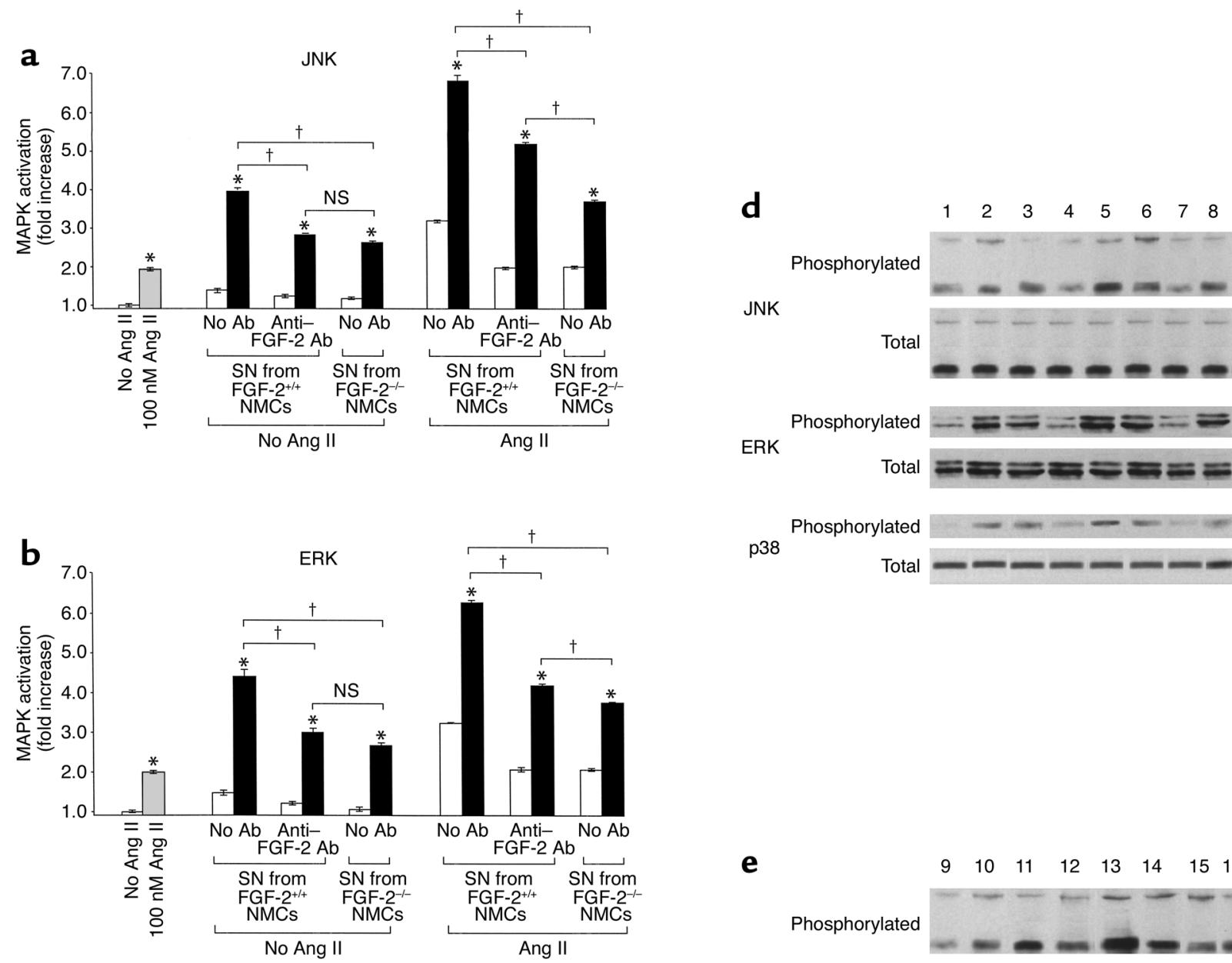

e
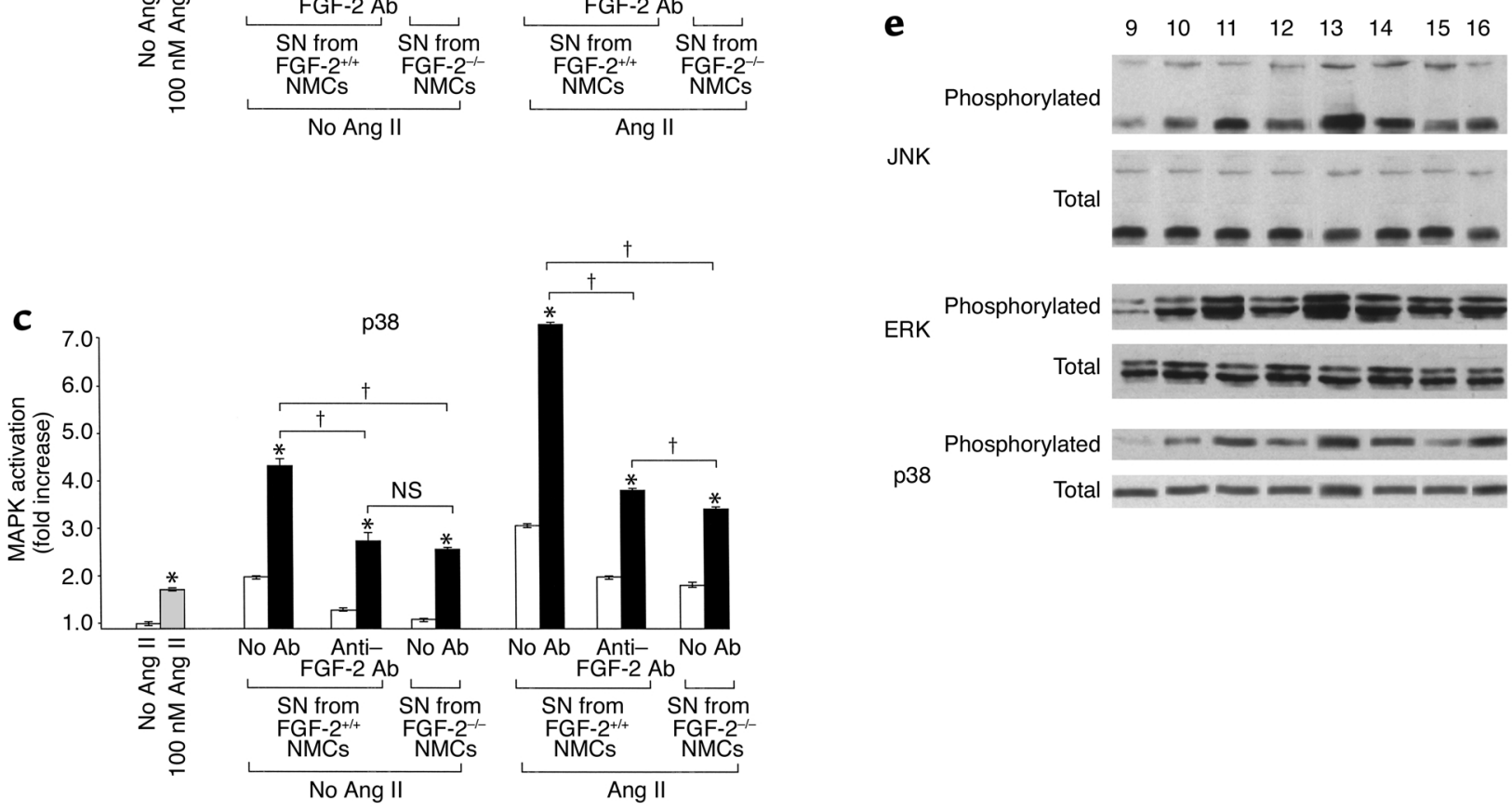

Figure 4

MAPK activation in primary cardiomyocytes by NMC culture supernatant. FGF-2 $2^{+/}$cardiomyocytes were unstimulated, or stimulated by 100 nM Ang II (gray bar), by supernatants (SN) from either unstimulated (white bars) or Ang II-stimulated NMC cultures (black bars), and by a combination of NMC SN and Ang II. Treatment was for 10 minutes for JNK (a) and 5 minutes for ERK (b) and p38 (c). Anti-FGF-2 Ab indicates addition of neutralizing antibodies to FGF-2. (d and e) Phosphorylation of JNK, ERK, and p38 was detected using specific antibodies. Lanes 1 and 9: unstimulated control; lanes 2 and 10: $100 \mathrm{nM} \mathrm{Ang} \mathrm{II;} \mathrm{lanes} \mathrm{3-8:} \mathrm{supernatants} \mathrm{from} \mathrm{unstimulated} \mathrm{NMCs;} \mathrm{lanes} \mathrm{11-16:}$ supernatants from Ang II-stimulated NMCs. Lanes 3-6 and 11-14: supernatants from FGF-2+/+ NMCs; lanes 7, 8, 15, and 16: supernatants from FGF-2-/- NMCs; lanes 5, 6, 8, 13, 14, and 16: $100 \mathrm{nM}$ Ang II present; lanes 4, 6, 12, and 14: anti-FGF-2 antibodies present. Data were quantified by laser scanning densitometry. Results represent fold activation compared with unstimulated control. Values are expressed as mean $\pm \operatorname{SEM}(n=3)$. ${ }^{*} P<0.05$ compared with unstimulated control. ${ }^{\dagger} P<0.05$. 

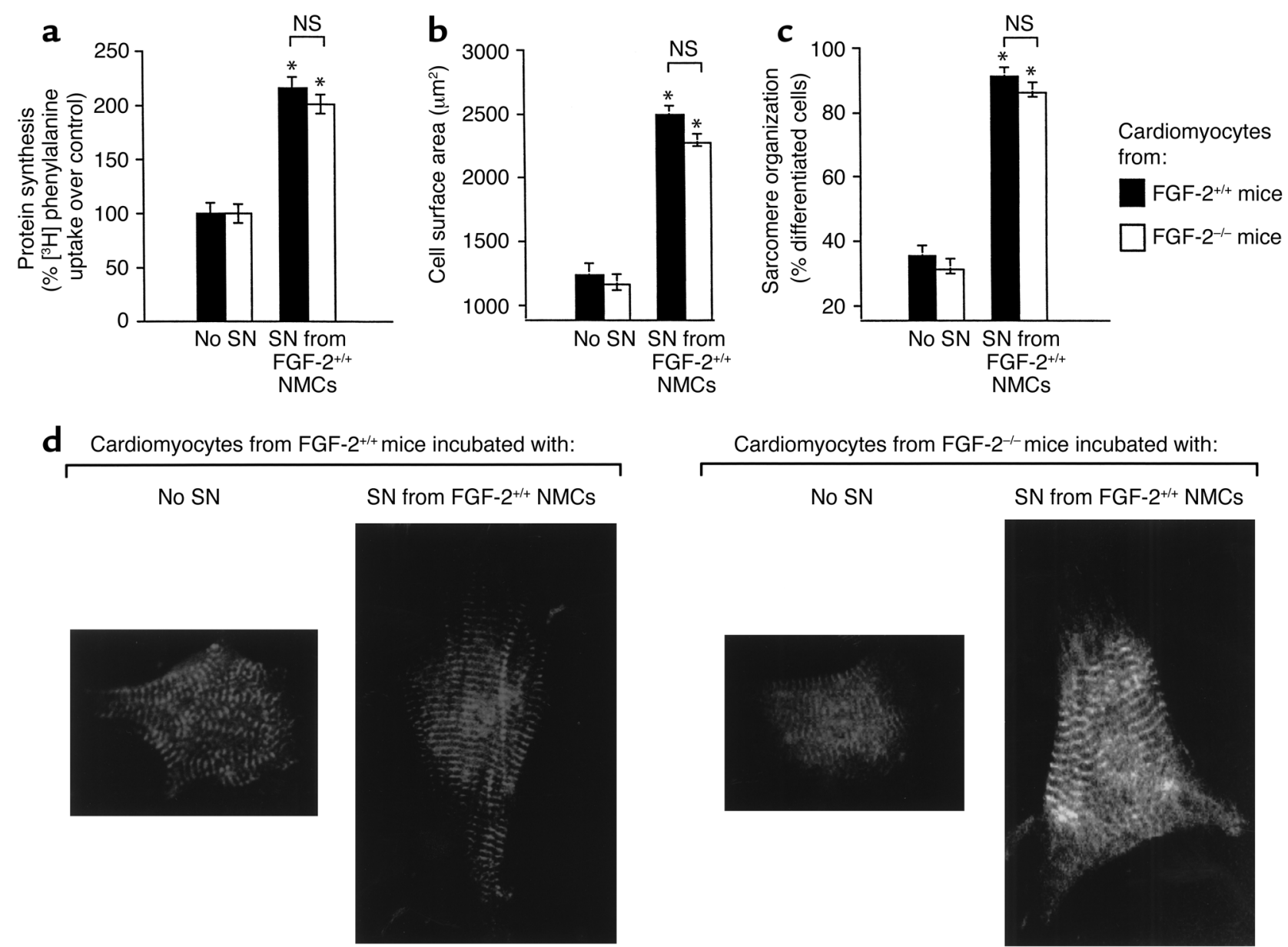

\section{Figure 5}

Normal hypertrophic response of FGF-2 $2^{+/}$and FGF-2-/- cardiomyocytes to supernatants from Ang II-stimulated FGF-2 ${ }^{+/+} \mathrm{NMCs}$. Cardiomyocytes from either FGF-2 ${ }^{+/+}$or FGF-2 $2^{-/-}$mice were stimulated with supernatants from FGF-2 ${ }^{+/+}$Ang II-stimulated NMC cultures. Protein synthesis was evaluated by $\left[{ }^{3} \mathrm{H}\right]$ phenylalanine incorporation (a). Fixed cells were stained with antibodies to myomesin $\mathrm{B} 4$, analyzed by confocal microscopy (d), and cell surface area $(\mathbf{b})$ and number of cells with organized sarcomeres $(\mathbf{c})$ were determined. ${ }^{*} P<0.05$ compared with unstimulated control.

Moreover, particularly in the case of JNK, the capacity of supernatants to stimulate MAPK after neutralization of FGF-2 activity was greater than that of supernatants from FGF-2-/- cultures. This suggests that the release of some factors is dependent on the ability of fibroblasts to secrete FGF-2.

Our results demonstrate that Ang II stimulates fibroblasts to produce growth factors. Several studies implicate Ang II in cardiac fibrosis due to stimulated fibroblast proliferation (29). Ang II also has direct growth-promoting effects on cardiomyocytes $(2,3)$. However, these effects could result in part from paracrine actions of growth factors from Ang II-stimulated fibroblasts. Indeed, inhibition of fibroblast proliferation in cultures of neonatal cardiac cells reduces Ang II-induced protein synthesis in cardiomyocytes (30). Furthermore, a recent study demonstrated the importance of AT-1-receptor expressing cardiomyocytes in the proliferative response of cardiac fibroblasts to Ang II (31). Indeed, Ang II, via AT-1 receptors, appears to upregulate FGF-2 expression in cardiac myocytes (7). FGF-2 itself increases FGF-2 transcription levels. Interestingly, culture supernatants from Ang II-activated cardiac fibroblasts stimulate protein synthesis in cardiomyocytes $(6,32)$ (Figure 5$)$. The activity of the supernatants is not blocked by antibodies directed against FGF-1 or FGF-2, but is significantly attenuated by the addition of neutralizing antibodies to TGF- $\beta$ (32). The results presented herein demonstrate the importance of FGF-2 in the stimulation of cardiomyocytes and in the release of other trophic factors by fibroblasts, and also the existence of extensive crosstalk between cardiac myocytes and fibroblasts that is crucial for the hypertrophic response.

Mice deficient in FGF-2 expression appear to suffer from dilated cardiomyopathy (Tables 1 and 2). Since cardiac dilation is already present in normotensive animals, it suggests that FGF-2 could play an important role in maintaining cardiac integrity. Recently, several groups independently generated mice that were deficient in FGF-2 expression (10, 20-22). Dilated cardiomyopathy in mice lacking FGF-2 has not been reported. The reason for this discrepancy is currently 

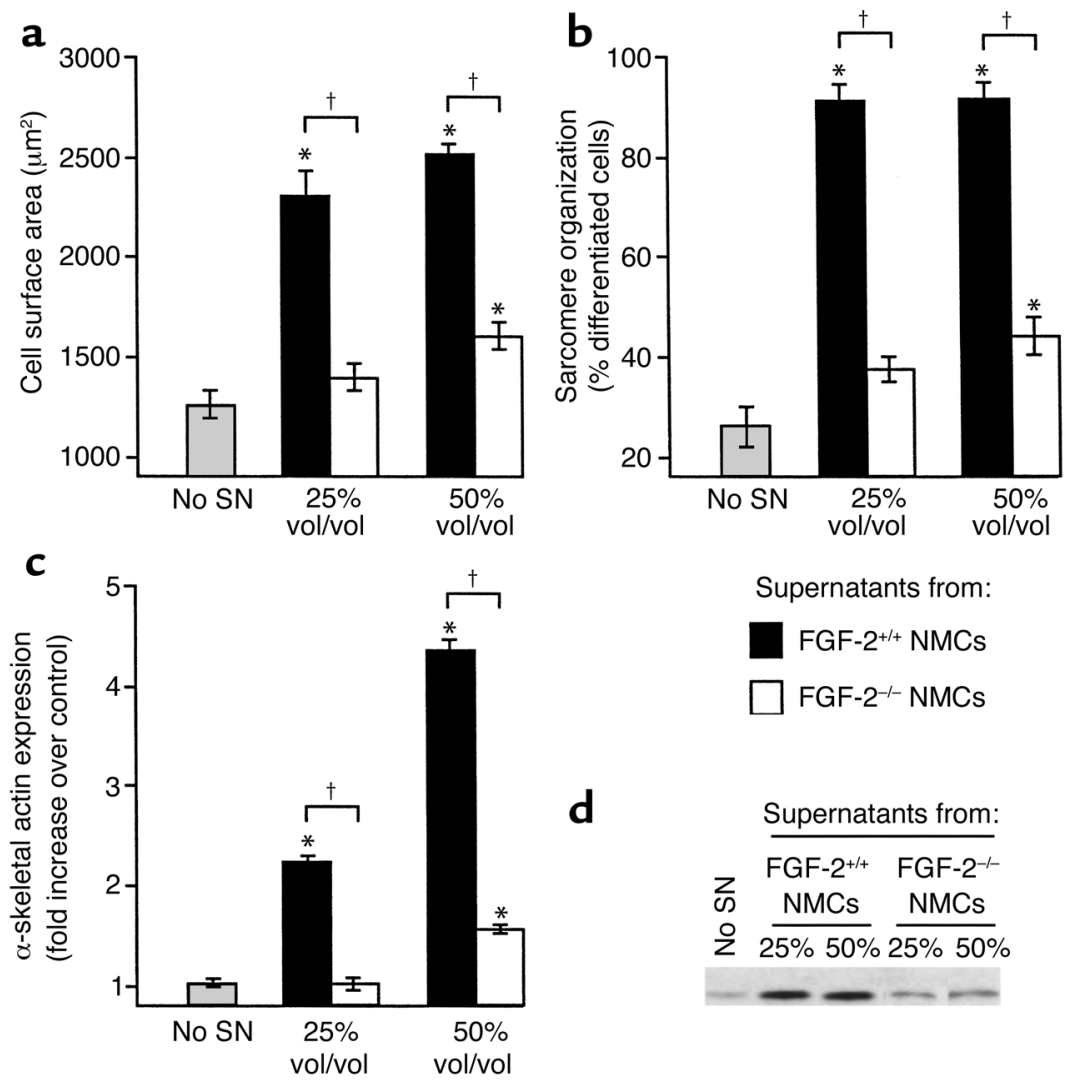

d

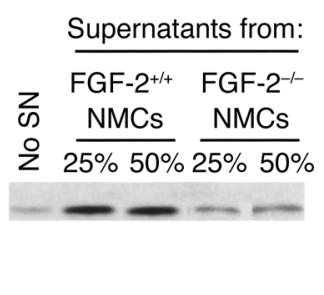

\section{Figure 6}

Deficient capacity of supernatants from FGF-2-/- NMCs to induce hypertrophic responses in $\mathrm{FGF}-2^{+/+}$cardiomyocytes. FGF-2+/+ cardiomyocytes were stimulated with supernatants from either $\mathrm{FGF}-2^{+/+} \mathrm{NMC}$ cultures (black bars) or FGF-2-/- NMC cultures (white bars), stained with antibodies to myomesin B4, and studied by confocal microscopy. Cell surface area (a) and number of cells exhibiting organized sarcomeres $(\mathbf{b})$ were determined. Expression of $\alpha$-skeletal actin was measured by Western blot analysis ( $c$ and $\mathbf{d}$ ). ${ }^{*} P<0.05$ compared with the unstimulated control. ${ }^{\dagger} P<0.05$.

not known. In the animals used in the present study, most of the second FGF-2 exon was replaced, and RTPCR analysis showed a total absence of FGF-2 expression (A. Foletti and F. Beermann, unpublished results). Similarly, FGF-2 message containing exon 2 has not been observed in earlier studies (21), therefore making it unlikely that some truncated forms of FGF-2 account for the different phenotype. Alternatively, the genetic background in which the animals are maintained could play a role. For instance, our mice were backcrossed in a one-renin-gene strain (24), whereas the mixed background of 129 and Black Swiss mice used in the previous studies carry two renin genes. In addition, one cannot exclude the possibility that distinct intracellular pathways are activated in response to pressure overload and Ang II-induced high blood pressure. In particular, we have recently demonstrated crosstalk between the MAPK, protein kinase $\mathrm{C}$, and calcineurin pathways in the Ang II-mediated hypertrophic response in renovascular hypertension (33).

Hypertensive animals lacking FGF-2 do not develop cardiac hypertrophy. Consistently, atrial natriuretic factor is not upregulated in the hearts of FGF-2-/- mice (Table 1 ). The absence of a hypertrophic response to chronic hypertension appears to result in part from a deficient production of growth factors from Ang II-activated cardiac fibroblasts. Indeed, cardiomyocytes fail to develop hypertrophy in vitro in response to supernatants from FGF-2-/- fibroblast cultures (Figure 6). In particular, $\alpha$-skeletal actin expression is not activated. FGF-2-deficient animals were previously shown to develop significantly less hypertrophy and reduced fibrosis in response to pressure overload (10). In this aortic coarctation model, the renin-angiotensin system is not activated, and plasma Ang II levels remain low. Thus, Ang II-induced stimulation of FGF-2 secretion probably did not play a major role. Indeed, pressure overload can induce cardiac hypertrophy in mice lacking AT- $1_{\mathrm{A}}$ receptors (34). Therefore, it is likely that hemodynamic overload represents the prime stimulator of FGF-2 release in this particular case, whereas in renovascular hypertension, both Ang II and Ang II-mediated increases in mechanical stress induce FGF-2 secretion from cardiac cells. FGF-2 lacks a signal sequence for secretion (5), and could exit the cells following stretch or injury. Mice lacking FGF-2 were shown to display some reduction in blood pressure but a normal blood pressure response to Ang II $(20,21)$. In the present study, FGF-2 knockouts are characterized by a nonsignificant reduction in blood pressure at basal conditions (Table 1). In addition, the elevation of blood pressure in the Ang II-dependent model of renovascular hypertension was not different from that seen in wild-type mice. This confirms a normal blood pressure response to Ang II in FGF-2 knockouts. On the other hand, Ang II directly contributes to hypertrophy $(2,3)$. Indeed, experiments in vitro demonstrated the capacity of Ang II to stimulate fibroblasts independently of hemodynamic stress. Our results suggest that various stimuli can activate fibroblasts in the heart. Thus, we propose that the cardiac fibroblast population may represent a primary integrator of biomechanical stress. Its activation would lead to growth factor secretion, and thereby to the induction of compensatory phenotypes in cardiomyocytes, provided that the hypertrophic stimulus is able to trigger FGF-2 release. 


\section{Acknowledgments}

The expert assistance of Thérèse Sauthier, Danièla Grand, and Irène Keller is gratefully acknowledged. We thank Jean-Yves Meuwly, Pierre Schnyder, and Alain Delabays for their help in the echographic assessment of cardiac performance. This work was supported in part by a grant from the Swiss National Research Foundation (32-61524.00, to T. Pedrazzini), and by a grant from the Swiss Cancer League (602-11998, to F. Beermann).

1.Page, I.H. 1949. Pathogenesis of arterial hypertension. JAMA. 140:451-465.

2. Sadoshima, J., and Izumo, S. 1993. Molecular characterization of angiotensin II-induced hypertrophy of cardiac myocytes and hyperplasia of cardiac fibroblasts. Critical role of the AT1 receptor subtype. Circ. Res. 73:413-423.

3. Dostal, D.E., et al. 1997. Molecular mechanisms of angiotensin II in modulating cardiac function: intracardiac effects and signal transduction pathways. J. Mol. Cell. Cardiol. 29:2893-2902.

4. Waeber, B., Nussberger, J., and Brunner, H.R. 1990. Angiotensin-converting-enzyme inhibitors in hypertension. In Hypertension: pathophysiol ogy, diagnosis, and management. J.H. Laragh and B.M. Brenner, editors. Raven Press Ltd. New York, New York, USA. 2209-2232.

5. Kardami, E., et al. 1995. Regulation of basic fibroblast growth factor (bFGF) and FGF receptors in the heart. Ann. NY Acad. Sci. 752:353-369.

6. Sil, P., and Sen, S. 1997. Angiotensin II and myocyte growth: role of fibroblasts. Hypertension. 30:209-216.

7. Fischer, T.A., et al. 1997. Regulation of bFGF expression and ANG II secretion in cardiac myocytes and microvascular endothelial cells. Am.J. Physiol. 272:H958-H968.

8. Kardami, E., and Fandrich, R.R. 1989. Basic fibroblast growth factor in atria and ventricles of the vertebrate heart. J. Cell Biol. 109:1865-1875.

9. Abramov, D., et al. 2000. Increased levels of basic fibroblast growth factor are found in the cross-clamped heart during cardiopulmonary bypass. Can. J. Cardiol. 16:313-318.

10. Schultz, J.E., et al. 1999. Fibroblast growth factor-2 mediates pressureinduced hypertrophic response. J. Clin. Invest. 104:709-719.

11. Parker, T.G., Parker, S.E., and Schneider, M.D. 1990. Peptide growth factors can provoke "fetal" contractile protein gene expression in rat cardiac myocytes. J. Clin. Invest. 85:507-514.

12. Parker, T.G., Chow, K.L., Schwartz, R.J., and Schneider, M.D. 1992. Positive and negative control of the skeletal alpha-actin promoter in cardiac muscle. J. Biol. Chem. 267:3343-3350.

13. Clarke, M.S.F., Caldwell, R.W., Chiao, H., Miyake, K., and McNeil, P.L 1995. Contraction-induced cell wounding and release of fibroblast growth factor in the heart. Circ. Res. 76:927-934.

14. Kaye, D., et al. 1996. Role of transiently altered sarcolemmal membrane permeability and basic fibroblast growth factor release in the hypertrophic response of adult rat ventricular myocytes to increased mechanical activity in vitro. J. Clin. Invest. 97:281-291.

15. Detillieux, K.A., Meij, J.T.A., Kardami, E., and Cattini, P.A. 1999. alpha1adrenergic stimulation of FGF-2 promoter in cardiac myocytes and in adult transgenic mouse hearts. Am. J. Physiol. 276:H806-H833.

16. Schaub, M.C., Hefti, M.A., Harder, B.A., and Eppenberger, H.M. 1997.
Various hypertrophic stimuli induce distinct phenotypes in cardiomyocytes. J. Mol. Med. 75:901-920.

17. Bogoyevitch, M.A., et al. 1994. Endothelin-1 and fibroblast growth factors stimulate the mitogen-activated protein kinase signaling cascade in cardiac myocytes. The potential role of the cascade in the integration of two signaling pathways leading to myocyte hypertrophy. J. Biol. Chem. 269:1110-1119.

18. McKinsey, T.A., and Olson, E.N. 1999. Cardiac hypertrophy: sorting out the circuitry. Curr. Opin. Genet. Dev. 9:267-274.

19. Jelinek, J., and Gross, F. 1970. Effects of differently induced renal ischaemia and of ureteral ligation on kidney renin and blood pressure. Cardiovasc. Res. 4:84-88.

20. Dono, R., Texido, G., Dussel, R., Ehmke, H., and Zeller, R. 1998. Impaired cerebral cortex development and blood pressure regulation in FGF-2deficient mice. EMBO J. 17:4213-4225.

21. Zhou, M., et al. 1998. Fibroblast growth factor 2 control of vascular tone. Nat. Med. 4:201-207.

22. Ortega, S., Ittmann, M., Tsang, S.H., Ehrlich, M., and Basilico, C. 1998. Neuronal defects and delayed wound healing in mice lacking fibroblast growth factor 2. Proc. Natl. Acad. Sci. USA. 95:5672-5677.

23. Wiesel, P., Mazzolai, L., Nussberger, J., and Pedrazzini, T. 1997. Two-kidney, one clip and one-kidney, one clip hypertension in mice. Hypertension. 29:1025-1030.

24. Field, L.J., and Gross, K.W. 1985. Ren-1 and Ren-2 loci are expressed in mouse kidney. Proc. Natl. Acad. Sci. USA. 82:6196-6200.

25. Helfman, D.M., et al. 1999. Nonmuscle tropomyosin-4 requires coexpression with other low molecular weight isoforms for binding to thin filaments in cardiomyocytes. J. Cell Sci. 112:371-380.

26. Clement, S., Chaponnier, C., and Gabbiani, G. 1999. A subpopulation of cardiomyocytes expressing alpha-skeletal actin is identified by a specific polyclonal antibody. Circ. Res. 85:e51-e58.

27. Pasumarthi, K.B.S., Kardami, E., and Cattini, P.A. 1996. High and low molecular weight fibroblast growth factor- 2 increase proliferation of neonatal rat cardiac myocytes but have differential effects on binucleation and nuclear morphology. Evidence for both paracrine and intracrine actions of fibroblast growth factor-2. Circ. Res. 78:126-136.

28. Corda, S., et al. 1997. Trophic effect of human pericardial fluid on adult cardiac myocytes. Differential role of fibroblast growth factor- 2 and factors related to ventricular hypertrophy. Circ. Res. 81:679-687.

29. Booz, G.W., and Baker, K.M. 1995. Molecular signalling mechanisms controlling growth and function of cardiac fibroblasts. Cardiovasc. Res. 30:537-543.

30. Kim, N.N., Villarreal, F.J., Printz, M., Lee, A.A., and Dillmann, W.H. 1995. Trophic effects of angiotensin II on neonatal rat cardiac myocytes are mediated by cardiac fibroblasts. Am. J. Physiol. 269:E426-E437.

31. Matsusaka, T., Katori, H., Inagami, T., Forgo, A., and Ichikawa, I. 1999. Communication between myocytes and fibroblasts in cardiac remodeling in angiotensin chimeric mice. J. Clin. Invest. 103:1451-1458.

32. Gray, O.G., Long, C.S., Kalinyak, J.E., Li, H., and Karliner, J.S. 1998. Angiotensin II stimulates cardiac myocyte hypertrophy via paracrine release of TGF-beta1 and endothelin-1 from fibroblasts. Cardiovasc. Res. 40:352-363.

33. Murat, A., Pellieux, C., Brunner, H.-R., and Pedrazzini, T. 2000. Calcineurin blockade prevents cardiac mitogen-activated protein kinase activation and hypertrophy in renovascular hypertension. J. Biol. Chem. 275:40867-40873.

34. Kudoh, S., et al. 1998. Mechanical stretch induces hypertrophic responses in cardiac myocytes of angiotensin II type 1a receptor knockout mice. J. Biol. Chem. 273:24037-24043. 